\title{
Utilising the radiative transfer equation in quantitative photoacoustic tomography
}

\author{
Tanja Tarvainen ${ }^{\mathrm{a}, \mathrm{b}}$, Aki Pulkkinen ${ }^{\mathrm{a}}$, Ben T. Cox ${ }^{\mathrm{c}}$, and Simon R. Arridge ${ }^{\mathrm{b}}$ \\ aDepartment of Applied Physics, University of Eastern Finland, P.O. Box 1627, FI-70211 \\ Kuopio, Finland \\ ${ }^{\mathrm{b}}$ Department of Computer Science, University College London, Gower Street, London WC1E \\ 6BT, UK \\ ${ }^{\mathrm{c}}$ Department of Medical Physics and Biomedical Engineering, University College London, \\ Gower Street, London WC1E 6BT, UK
}

\begin{abstract}
Quantitative photoacoustic tomography is an emerging imaging technique aimed at estimating optical parameters inside tissue from photoacoustic images. This optical parameter estimation problem is an ill-posed inverse problem, and thus it is sensitive to measurement and modelling errors. Therefore, light propagation in quantitative photoacoustic tomography needs to be accurately modelled. A widely accepted model for light propagation in biological tissue is the radiative transfer equation. In this work, the radiative transfer equation is utilised in quantitative photoacoustic tomography. Estimating absorption and scattering distributions in quantitative photoacoustic tomography using various illuminations is investigated.
\end{abstract}

Keywords: Quantitative photoacoustic tomography, radiative transfer equation, inverse problems

\section{INTRODUCTION}

Quantitative photoacoustic tomography (QPAT) is an emerging imaging technique aimed at estimating the optical parameters inside tissue from photoacoustic images which are formed by combining optical information and ultrasonic propagation. ${ }^{1}$ Thus, the method proceeds from photoacoustic tomography by taking the estimated initial pressure distributions as data and estimating the absolute values of the optical parameters.

The two inverse problems of QPAT are: 1) reconstruct the initial acoustic pressure distribution from measured acoustic waves and 2) reconstruct the distributions of the optical parameters from the absorbed optical energy density. The first inverse problem is an inverse initial value problem of acoustics. There are a large number of reconstruction techniques available, see e.g. ${ }^{2-4}$ and the references therein. However, in cases in which the speed of sound, acoustic absorption and scattering within the tissue are varying, the inverse problem becomes significantly more challenging.

The second inverse problem in QPAT is the optical image reconstruction. In the optical inverse problem, the goal is to estimate the concentrations of chromophores. These can be obtained either by directly estimating the chromophore concentrations from photoacoustic images obtained at various wavelengths ${ }^{5-8}$ or by first recovering the absorption coefficients at different wavelengths and then calculating the concentrations from the absorption spectra..$^{5,7,9}$ In order to obtain accurate estimates, scattering effects need to be taken into account. ${ }^{1,10-12}$ As an alternative to the two-step approach to the solution of the QPAT inverse problem, estimation of the optical parameters directly from photoacoustic time series has also been considered recently. ${ }^{13-18}$

In this work, the optical inverse problem of QPAT is considered. The optical parameter estimation problem is ill-posed, and thus it is sensitive to measurement and modelling errors. Therefore, light propagation and absorption in QPAT needs to be accurately modelled. A widely accepted model for light propagation in biological tissue is the radiative transfer equation (RTE) ${ }^{19,20}$ However, it is computationally expensive, and therefore its

Further author information: (Send correspondence to T.T.)

T.T.: E-mail: tanja.tarvainen@uef.fi, Telephone: +358 403552310

Photons Plus Ultrasound: Imaging and Sensing 2017, edited by Alexander A. Oraevsky, Lihong V. Wang, Proc. of SPIE Vol. 10064, 100643E · (c) 2017 SPIE · CCC code: 1605-7422/17/\$18 · doi: 10.1117/12.2249310 
approximation in strongly scattering medium, the diffusion approximation (DA), is often utilised in biomedical imaging. ${ }^{20}$ In QPAT, however, imaging depth can be small compared to the average scattering length, and thus the DA is not a valid approximation. ${ }^{19,20}$

Utilising the RTE in QPAT has previously been considered in Refs. ${ }^{11,21-25}$ Comparison of the performance of the RTE and the DA as light transport models in the optical inverse problem of QPAT was considered in Ref. ${ }^{11}$ Furthermore, a gradient based method for the problem was developed in Ref. ${ }^{23}$ In, ${ }^{25}$ the RTE was utilised in QPAT in the case of using illuminations from a single direction. In this work, this approach is further investigated. Estimation of absorption and scattering is considered and reconstructions obtained using the RTE and DA as the light transport models are compared.

\section{METHODS}

The optical inverse problem in QPAT is to estimate the optical properties of the object when the absorbed optical energy density $H$ is given. In this work, the absorption $\mu_{a}$ and scattering $\mu_{s}$ distributions are estimated by minimising the functional

$$
\left(\hat{\mu}_{a}, \hat{\mu}_{s}\right)=\underset{\mu_{a}, \mu_{s}}{\arg \min }\left\{\left\|L_{e}\left(H_{\text {meas }}-H\left(\mu_{a}, \mu_{s}\right)\right)\right\|^{2}+\left\|L_{\mu_{a}}\left(\mu_{a}-\eta_{\mu_{a}}\right)\right\|^{2}+\left\|L_{\mu_{s}}\left(\mu_{s}-\eta_{\mu_{s}}\right)\right\|^{2}\right\}
$$

where $H_{\text {meas }}$ and $H$ are the measured and modelled absorbed optical energy density. Further, matrix $L_{e}$ is the Cholesky decomposition of the inverse of the noise covariance matrix $\Gamma_{e}^{-1}=L_{e}^{\mathrm{T}} L_{e}, \eta_{\mu_{a}}$ and $\eta_{\mu_{s}}$ are the means of the prior for absorption and scattering, respectively, and $L_{\mu_{a}}$ and $L_{\mu_{s}}$ are the Cholesky decompositions of the inverse of the prior covariance matrices for absorption and scattering $\Gamma_{\mu_{a}}^{-1}=L_{\mu_{a}}^{\mathrm{T}} L_{\mu_{a}}$ and $\Gamma_{\mu_{s}}^{-1}=L_{\mu_{s}}^{\mathrm{T}} L_{\mu_{s}}$. In this work, an informative smoothness prior is utilised. ${ }^{26,27}$

The minimisation problem (1) is solved using a Gauss-Newton method with a line search algorithm, for determination of the step length, and a positivity constraint for the estimated parameters. ${ }^{11,28}$

\subsection{Forward Model}

A widely accepted model for light transport in tissues is the radiative transfer equation. ${ }^{19,20}$ The timeindependent RTE together with a boundary condition is of the form

$$
\begin{aligned}
& \hat{s} \cdot \nabla \phi(r, \hat{s})+\left(\mu_{a}+\mu_{s}\right) \phi(r, \hat{s})=\mu_{s} \int_{S^{n-1}} \Theta\left(\hat{s} \cdot \hat{s}^{\prime}\right) \phi\left(r, \hat{s}^{\prime}\right) \mathrm{d} \hat{s}^{\prime}, \quad r \in \Omega \\
& \phi(r, \hat{s})=\left\{\begin{array}{cl}
\phi_{0}(r, \hat{s}), & r \in \epsilon_{j}, \quad \hat{s} \cdot \hat{n}<0 \\
0, & r \in \partial \Omega \backslash \epsilon_{j}, \quad \hat{s} \cdot \hat{n}<0
\end{array}\right.
\end{aligned}
$$

where $\Omega \subset \mathbb{R}^{n},(n=2$ or 3$)$ is the domain with boundary $\partial \Omega, \hat{s} \in S^{n-1}$ is the unit vector in the direction of interest, $\phi(r, \hat{s})$ is the radiance, $\Theta\left(\hat{s} \cdot \hat{s}^{\prime}\right)$ is the scattering phase function, and $\phi_{0}(r, \hat{s})$ is an inward directed light source on the boundary $\epsilon_{j} \subset \partial \Omega$. The absorbed optical energy density $H(r)$, can be solved as

$$
H(r)=\mu_{a} \Phi(r)
$$

where $\Phi(r)$ is the photon fluence

$$
\Phi(r)=\int_{S^{n-1}} \phi(r, \hat{s}) \mathrm{d} \hat{s}
$$

In the diffusion approximation framework, the radiance is approximated by

$$
\phi(r, \hat{s}) \approx \frac{1}{\left|S^{n-1}\right|} \Phi(r)-\frac{n}{\left|S^{n-1}\right|} \hat{s} \cdot(\kappa \nabla \Phi(r))
$$

where $\kappa=\left(n\left(\mu_{a}+\mu_{s}^{\prime}\right)\right)^{-1}$ is the diffusion coefficient where $\mu_{s}^{\prime}=(1-g) \mu_{s}$ is the reduced scattering coefficient and $g$ is the mean of the cosine of the scattering angle. ${ }^{20,29}$ By inserting the approximation (6) and similar 
approximations written for the source term and phase function into equation (2) and following the derivation in, ${ }^{19,20,30}$ we obtain the DA which, together with a Robin-type boundary condition, can be written as

$$
\begin{aligned}
& -\nabla \cdot \kappa \nabla \Phi(r)+\mu_{a} \Phi(r)=0, \quad r \in \Omega \\
& \Phi(r)+\frac{1}{2 \gamma_{n}} \kappa \frac{\partial \Phi(r)}{\partial \hat{n}}= \begin{cases}\frac{I_{s}}{\gamma_{n}}, & r \in \epsilon_{j} \\
0, & r \in \partial \Omega \backslash \epsilon_{j}\end{cases}
\end{aligned}
$$

where $I_{s}$ is a diffuse boundary current at the source position $\epsilon_{j} \subset \partial \Omega$ and $\gamma_{n}$ is a dimension-dependent constant which takes values $\gamma_{2}=1 / \pi$ and $\gamma_{3}=1 / 4$. Basically, the approximation (6) means that the radiance is assumed to be almost uniform with respect to angular direction. This is generally valid in turbid media sufficiently far from light sources and boundaries. ${ }^{19,20}$

In this work, the RTE and the DA are numerically solved using the finite element method (FEM) ${ }^{30,31}$ In the FE-approximation of the RTE both spatial and angular discretisation are implemented in piecewise linear bases. Furthermore, a streamline diffusion modification is utilised. ${ }^{32,33}$

\section{RESULTS}

The optical inverse problem of QPAT was investigated with two-dimensional (2D) simulations. A rectangular domain of size $4 \mathrm{~mm} \times 8 \mathrm{~mm}$ was considered. In a domain of this size, the approximations of the DA are not valid and the RTE is a more accurate light transport model.

Two types of imaging situations were considered: an approach where the target was illuminated from various directions and an approach in which the target was illuminated from one direction. In the inverse problem of QPAT, in the absence of other suitable prior information, simultaneous estimation of both absorption and scattering is generally non-unique if only one light illumination is used. ${ }^{10,24}$ In this work, spatially modulated light was utilised to overcome the non-uniqueness problem in the case of illuminations from one direction. ${ }^{25}$ The different illuminations that were utilised are shown in Fig. 1.

The simulation domain included varying and partially overlapping absorption and scattering inclusions. The distributions of simulated absorption and scattering values are shown on the top row of Figs. 2 and 3. The anisotropy parameter was $g=0.8$ throughout the domain. The data was simulated using the RTE (2)-(3). The spatial FE-discretisation consisted of 9322 triangular elements and 4782 nodes. Furthermore, 128 angular directions were used. Gaussian distributed noise with standard deviation of $1 \%$ of the amplitude was added into the simulated data. Three types of data was simulated: i) data using four planar illuminations from different directions, ii) data using four light patterns illuminated from top of the target and iii) data using one planar illumination from top of the target.

The absorption and scattering distributions were reconstructed by minimising (1). Both the RTE (2)-(3) and the DA (7)-(8) were used as light transport model. The FE-element discretisation consisted of 1308 triangular elements and 703 nodes. In the case of the RTE, 64 angular directions were used. The reconstructed absorption and scattering distributions obtained using the RTE as light transport model are shown in Fig. 2 and the reconstructed absorption and scattering distributions obtained using the DA as light transport model are shown in Fig. 3.

The results show that the best quality reconstructions are obtained by utilising illuminations from various directions and the RTE as light transport model. In that case, both absorbing and scattering inclusions can be reconstructed. In the case of illuminating the target only from one direction using spatially modulated light and the RTE as light transport model, the absorption images are almost of the same quality as in the case of multi-direction illuminations. Only the inclusions located furthest from the illuminating edge show weaker reconstructed absorption values. Information on scattering is, however, quickly lost as the distance to the illumination edge increases.

In the case of the reconstructions obtained using the DA as the light transport model, the reconstructed absorption distributions appear to be of the same quality as the RTE reconstructions. The reconstructed scattering distributions are, however, significantly poorer on quality and the inclusions can be distinguished only in 

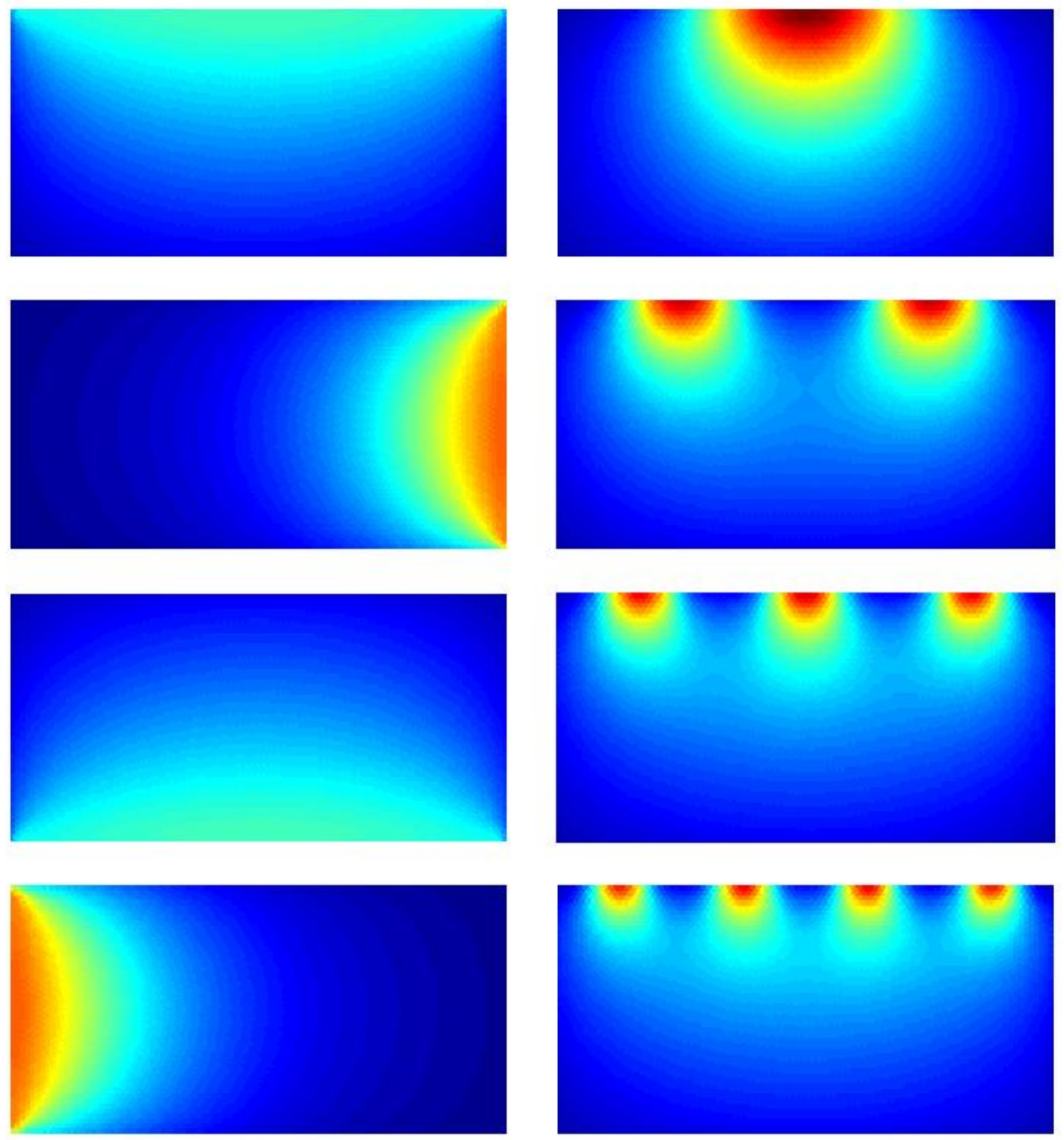

Figure 1. Fluence created by different illuminations used in the simulations. Four illuminations from different directions (left column) and four spatially modulated light patterns at one side of the target (right column).

the case of multiple illuminations from various directions. However, also in that case, the contrast of scattering is much weaker compared to scattering reconstructions obtained using the RTE.

If only one planar illumination is used, both the RTE and DA give unreliable estimates for absorption and scattering. This is due to ill-posedness and non-uniqueness of the problem.

\section{CONCLUSIONS}

In this work, estimating absorption and scattering distributions in QPAT using multiple illuminations was investigated. Planar illuminations from various directions and spatially modulated light from one direction were investigated. The RTE was used as light transport model and the results were compared to reconstructions obtained using the DA as light transport model. The results show that, if the target can be illuminated only from one direction, utilising spatially modulated light can significantly improve the reconstructions compared 

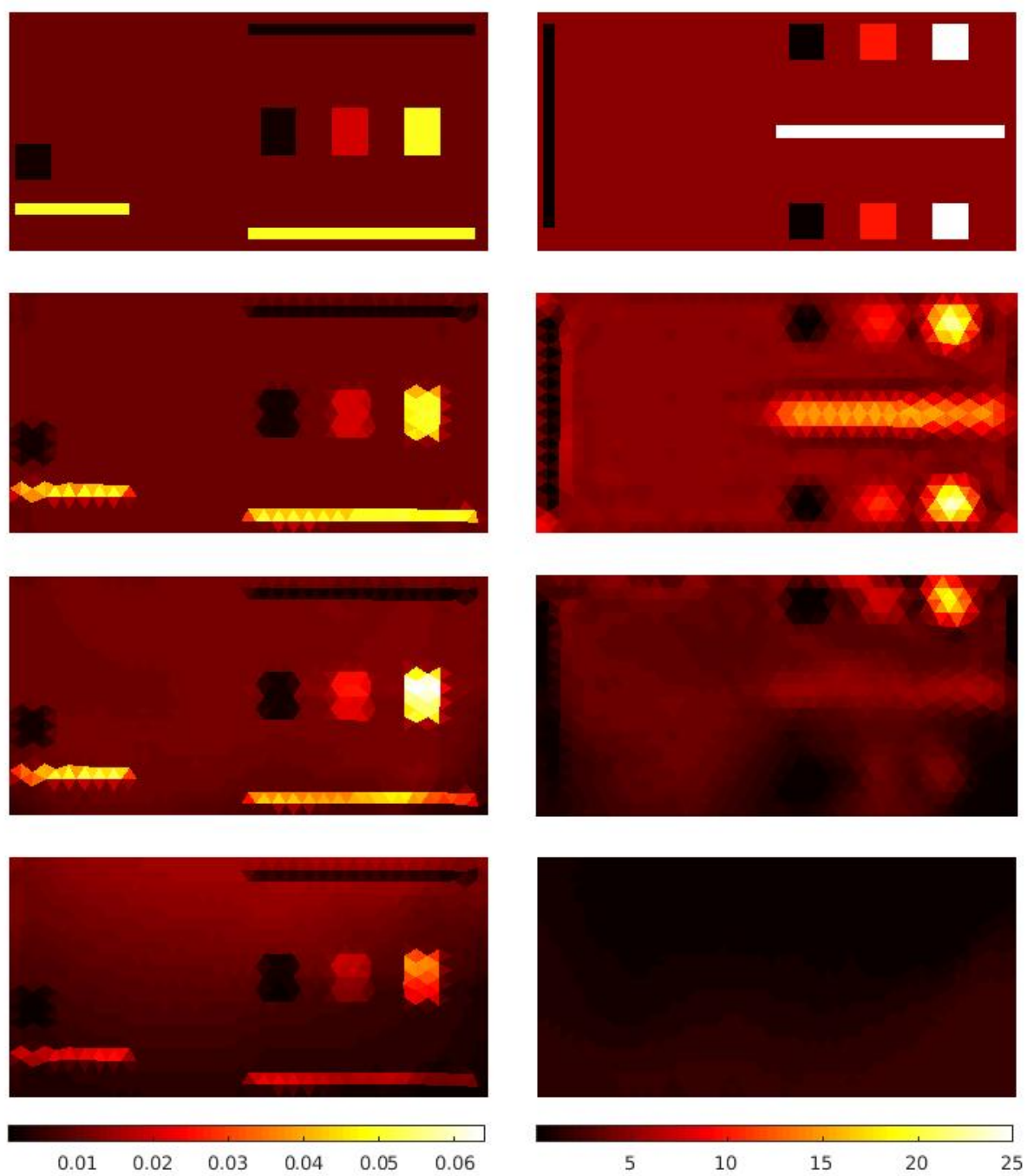

Figure 2. Reconstructed absorption (left column) and scattering (right column) distributions obtained using the RTE as the light transport model. Images from top to bottom: simulated distributions (first row), reconstructions obtained using planar illuminations from various directions (second row), reconstructions obtained using spatially modulated illumination patterns (third row) and reconstructions obtained using one planar illumination (fourth row). The units of the colorbars are in $\mathrm{mm}^{-1}$.

to one planar illumination. However, the quality of the scattering images is comparable to the quality of the reconstructions obtained using multi-direction illuminations only close to the illuminating edge.

\section{ACKNOWLEDGMENTS}

This work has been supported by the Academy of Finland (projects 286247 and 250215 Finnish Centre of Excellence in Inverse Problems Research), Magnus Ehrnrooth Foundation, and Jane and Aatos Erkko Foundation. 

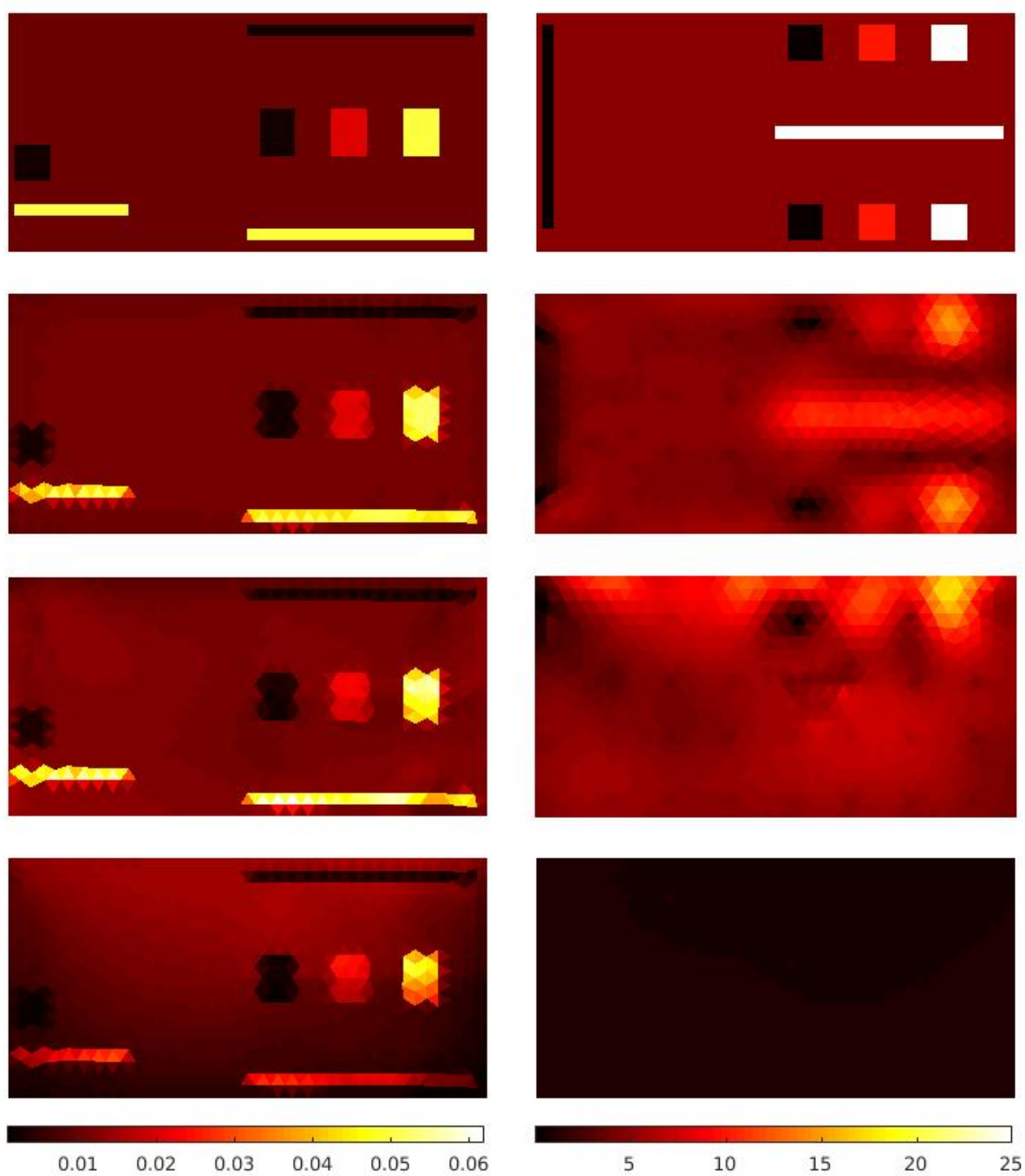

Figure 3. Reconstructed absorption (left column) and scattering (right column) distributions using the DA as the light transport model. Images from top to bottom: simulated distributions (first row), reconstructions obtained using planar illuminations from various directions (second row), reconstructions obtained using spatially modulated illumination patterns (third row) and reconstructions obtained using one planar illumination (fourth row). The units of the colorbars are in $\mathrm{mm}^{-1}$.

\section{REFERENCES}

[1] Cox, B., Laufer, J. G., Arridge, S. R., and Beard, P. C., "Quantitative spectroscopic photoacoustic imgaging: a review," J Biomed Opt 17(6), 061202 (2012).

[2] Xu, M. and Wang, L. V., "Photoacoustic imaging in biomedicine," Rev Sci Instrum 77, 041101 (2006).

[3] Kuchment, P. and Kunyansky, L., "Mathematics of thermoacoustic tomography," European Journal of Applied Mathematics 19, 191-224 (2008).

[4] Wang, L. V., ed., [Photoacoustic Imaging and Spectroscopy], CRC Press (2009).

[5] Cox, B. T., R., S., Arridge, and Beard, P. C., "Estimating chromophore distributions from multiwavelength photoacoustic images," J Opt Soc Am A 26(2), 443-455 (2009). 
[6] Laufer, J., Cox, B., Zhang, E., and Beard, P., "Quantitative determination of chromophore concentrations form 2D photoacoustic images using a nonlinear model-based inversion scheme," Appl Opt 49(8), 1219-1233 (2010).

[7] Bal, G. and Ren, K., "On multi-spectral quantitative photoacoustic tomography in a diffusive regime," Inv Probl 28, 025010 (2012).

[8] Pulkkinen, A., Cox, B. T., Arridge, S. R., Kaipio, J. P., and Tarvainen, T., "A Bayesian approach to spectral quantitative photoacoustic tomography," Inv Probl 30, 065012 (2014).

[9] Razansky, D., Baeten, J., and Ntziachristos, V., "Sensitivity of molecular target detection by multispectral optoacoustic tomography (MSOT)," Med Phys 36(3), 939-945 (2009).

[10] Bal, G. and Ren, K., "Multi-source quantitative photoacoustic tomography in a diffusive regime," Inv Probl 27, 075003 (2011).

[11] Tarvainen, T., Cox, B. T., Kaipio, J. P., and Arridge, S. R., "Reconstructing absorption and scattering distributions in quantitative photoacoustic tomography," Inv Probl 28, 084009 (2012).

[12] Pulkkinen, A., Kolehmainen, V., Kaipio, J. P., Cox, B. T., Arridge, S. R., and Tarvainen, T., "Approximate marginalization of unknown scattering in quantitative photoacoustic tomography," Inv Probl Imag 8(3), 811-829 (2014).

[13] Shao, P., Harrison, T., and Zemp, R. J., "Iterative algorithm for multiple illumination photoacoustic tomography (MIPAT) using ultrasound channel data," Biomed Opt Express 3(12), 3240-3248 (2012).

[14] Song, N., Deumié, C., and Silva, A. D., "Considering sources and detectors distributions for quantitative photoacoustic tomography," Biomed Opt Express 5(11), 3960-3974 (2014).

[15] Haltmeier, M., Neumann, L., and Rabanser, S., "Single-stage reconstruction algorithm for quantitative photoacoustic tomography," Inv Probl 31, 065005 (2015).

[16] Gao, H., Feng, J., and Song, L., "Limited-view multi-source quantitative photoacoustic tomography," Inv Probl 31, 065004 (2015).

[17] Ding, T., Ren, K., and Vallélian, S., "A one-step reconstruction algorithm for quantitative photoacoustic imaging," Inv Probl 31, 095005 (2015).

[18] Pulkkinen, A., Cox, B. T., Arridge, S. R., Goh, H., Kaipio, J. P., and Tarvainen, T., "Direct estimation of optical parameters from photoacoustic time series in quantitative photoacoustic tomography," IEEE Trans Med Imag 35(11), 2497-2508 (2016).

[19] Ishimaru, A., [Wave Propagation and Scattering in Random Media], vol. 1, Academic Press, New York (1978).

[20] Arridge, S. R., "Optical tomography in medical imaging," Inv Probl 15, R41-R93 (1999).

[21] Yao, L., Sun, Y., and Jiang, H., "Quantitative photoacoustic tomography based on the radiative transfer equation," 34(12), 1765-1767 (2009).

[22] Yao, L., Sun, Y., and Jiang, H., "Transport-based quantitative photoacoustic tomography: simulations and experiments," Phys Med Biol 55, 1917-1934 (2010).

[23] Saratoon, T., Tarvainen, T., Cox, B. T., and Arridge, S. R., "A gradient-based method for quantitative photoacoustic tomography using the radiative transfer equation," Inv Probl 29, 075006 (2013).

[24] Mamonov, A. V. and Ren, K., "Quantitative photoacoustic imaging in radiative transport regime," Comm. Math. Sci 12, 201-234 (2014).

[25] Pulkkinen, A., Cox, B. T., Arridge, S. R., Kaipio, J. P., and Tarvainen, T., "Quantitative photoacoustic tomography using illuminations from a single direction," J Biomed Opt 20(3), 036015 (2015).

[26] Arridge, S. R., Kaipio, J. P., Kolehmainen, V., Schweiger, M., Somersalo, E., Tarvainen, T., and Vauhkonen, M., "Approximation errors and model reduction with an application in optical diffusion tomography," Inv Probl 22, 175-195 (2006).

[27] Kolehmainen, V., Schweiger, M., Nissilä, I., Tarvainen, T., Arridge, S. R., and Kaipio, J. P., "Approximation errors and model reduction in three-dimensional diffuse optical tomography," J Opt Soc Am A 26(10), 22572268 (2009).

[28] Tarvainen, T., Pulkkinen, A., Cox, B. T., Kaipio, J. P., and Arridge, S. R., "Bayesian image reconstruction in quantitative photoacoustic tomography," IEEE Trans Med Imag 32(12), 2287-2298 (2013).

[29] Kaipio, J. and Somersalo, E., [Statistical and Computational Inverse Problems], Springer, New York (2005). 
[30] Tarvainen, T., Computational Methods for Light Transport in Optical Tomography, PhD thesis, University of Kuopio, Kuopio, Finland (2006).

[31] Tarvainen, T., Vauhkonen, M., Kolehmainen, V., and Kaipio, J. P., "Hybrid radiative-transfer-diffusion model for optical tomography," Appl Opt 44(6), 876-886 (2005).

[32] Kanschat, G., "A robust finite element discretization for radiative transfer problems with scattering," EastWest J. Numer. Math 6(4), 265-272 (1998).

[33] Tarvainen, T., Vauhkonen, M., Kolehmainen, V., Arridge, S., and Kaipio, J., "Coupled radiative transfer equation and diffusion approximation model for photon migration in turbid medium with low-scattering and non-scattering regions," Phys Med Biol 50, 4913-4930 (2005). 